\title{
Electronic retrieval of health information by healthcare providers to improve practice and patient care (Review)
}

McGowan J, Grad R, Pluye P, Hannes K, Deane K, Labrecque M, Welch V, Tugwell P

McGowan J, Grad R, Pluye P, Hannes K, Deane K, Labrecque M, Welch V, Tugwell P.

Electronic retrieval of health information by healthcare providers to improve practice and patient care.

Cochrane Database of Systematic Reviews 2009, Issue 3. Art. No.: CD004749.

DOI: 10.1002/14651858.CD004749.pub2.

www.cochranelibrary.com

Electronic retrieval of health information by healthcare providers to improve practice and patient care (Review) Copyright $\odot 2010$ The Cochrane Collaboration. Published by John Wiley \& Sons, Ltd. 
TABLE OF CONTENTS

HEADER 1

ABSTRACT

PLAIN LANGUAGE SUMMARY

BACKGROUND

OBJECTIVES

METHODS

RESULTS

Figure 1.

DISCUSSION

AUTHORS' CONCLUSIONS

ACKNOWLEDGEMENTS

REFERENCES

CHARACTERISTICS OF STUDIES

ADDITIONAL TABLES

APPENDICES

WHAT'S NEW

HISTORY

CONTRIBUTIONS OF AUTHORS

DECLARATIONS OF INTEREST

SOURCES OF SUPPORT 
[Intervention Review]

\section{Electronic retrieval of health information by healthcare providers to improve practice and patient care}

Jessie McGowan¹, Roland Grad², Pierre Pluye³ ${ }^{3}$ Karin Hannes 4 , Katherine Deane ${ }^{5}$, Michel Labrecque ${ }^{6}$, Vivian Welch 7 , Peter Tugwell8

1Institute of Population Health/Ottawa Health Research Institute, University of Ottawa, Ottawa, Canada. 2Family Medicine, McGill University, Montreal, Canada. ${ }^{3}$ Département de médecine familiale, Université McGill, Montréal, Canada. ${ }^{4}$ Belgian Centre for EvidenceBased Medicine and Joanna Briggs Institute Australia, Leuven, Belgium. ${ }^{5}$ Edith Cavell Building, University of East Anglia, Norwich, UK. ${ }^{6}$ Département de médecine familiale, Université Laval, Centre hospitalier universitaire de Québec - Hôpital St-François d'Assise, Québec, Canada. ${ }^{7}$ Centre for Global Health, Institute of Population Health, University of Ottawa, Ottawa, Canada. ${ }^{8}$ Centre for Global Health, Institute of Population Health, Department of Medicine, University of Ottawa, Ottawa, Canada

Contact address: Jessie McGowan, Institute of Population Health/Ottawa Health Research Institute, University of Ottawa, 1 Stewart St. room 206, Ottawa, Ontario, K1N 6N5, Canada.jmcgowan@uottawa.ca.

Editorial group: Cochrane Effective Practice and Organisation of Care Group.

Publication status and date: Edited (no change to conclusions), published in Issue 3, 2010.

Citation: McGowan J, Grad R, Pluye P, Hannes K, Deane K, Labrecque M, Welch V, Tugwell P. Electronic retrieval of health information by healthcare providers to improve practice and patient care. Cochrane Database of Systematic Reviews 2009, Issue 3. Art. No.: CD004749. DOI: 10.1002/14651858.CD004749.pub2.

Copyright ( 2010 The Cochrane Collaboration. Published by John Wiley \& Sons, Ltd.

\section{A B S T R A C T}

\section{Background}

The movement towards evidence-based practice makes explicit the need for access to current best evidence to improve health. Advances in electronic technologies have made health information more available, but does availability affect the rate of use of evidence in practice?

\section{Objectives}

To assess the effectiveness of interventions intended to provide electronic retrieval (access to information) to health information by healthcare providers to improve practice and patient care.

\section{Search methods}

We obtained studies from computerized searches of multiple electronic bibliographic databases, supplemented by checking reference lists, and consultation with experts.

\section{Selection criteria}

Randomized controlled trials (RCTs) including cluster randomized trials (CRCTs), controlled clinical trials (CCT), and interrupted time series analyses (ITS) of any language publication status examining interventions of effectiveness of electronic retrieval of health information by healthcare providers.

\section{Data collection and analysis}

Duplicate relevancy screening of searches, data abstraction and risk of bias assessment was undertaken.

\section{Main results}

We found two studies that examined this question. Neither study found any changes in professional behavior following an intervention that facilitated electronic retrieval of health information. There was some evidence of improvements in knowledge about the electronic sources of information reported in one study. Neither study assessed changes in patient outcomes or the costs of provision of the electronic resource and the implementation of the recommended evidence-based practices. 


\section{Authors' conclusions}

Overall there was insufficient evidence to support or refute the use of electronic retrieval of healthcare information by healthcare providers to improve practice and patient care.

\section{PLAIN LANGUAGE SUMMARY}

\section{Electronic retrieval of health information by health professionals}

This summary of a Cochrane review presents what we know from research about the effect of health professionals' access to health information on patient care.

\section{What is electronic access to health information and who are health professionals?}

Healthcare professionals need access to the latest research to help them take care of their patients. Health information means research evidence from a reliable source. Electronic access to the latest health information means being able to find and read articles and other material using a computer and an Internet connection. Health professionals might read this information on the computer screen or in paper form. They may have training on how to find health information or they may not.

Health professionals are doctors, nurses, pharmacists, physiotherapists, occupational therapists, dietitians, nutritionists or anyone else who takes care of patients.

\section{The review shows that;}

- There was no difference in the practice of health professionals in hospitals that provided training to health professionals in finding information from the Cochrane Reproductive Health Library (a reliable source of information) and those that did not provide training.

- Knowledge of the Cochrane Reproductive Health Library was better among health care professionals that had training.

- We do not know the effects of electronic access to information on patient care, nor how much it cost to provide and implement the access because this information was not reported in the studies included in this review.

- More research is needed to determine the best methods for providing electronic access and training to health professionals in order to improve patient care. 


\section{B A C K G R O U N D}

The movement towards evidence-based practice makes the explicit need for access to current best evidence to improve health. The ability to improve health is dependent on our understanding of its determinants and the application of that knowledge in the prevention and treatment of disease. This understanding in turn depends on the dissemination and organization of research findings in ways that build on and make sense of existing information (Pakenham-Walsh 2002). Knowledge access had been suggested as potentially the single most cost-effective and achievable strategy for improvement of health care (PakenhamWalsh 1997).

Historically, healthcare providers have accessed a variety of information resources to inform their decision-making. Traditional resources of health information for healthcare professionals included printed materials, such as textbooks, clinical manuals, journals and drug reference books (Dawes 2003). According to a systematic literature review, the dissemination of printed education materials (PEMs) of research results published in healthcare journals and printed clinical practice guidelines has small beneficial effects on professional practice (Farmer 2008). When compared to no intervention, PEMs when used alone may have a beneficial effect on process outcomes, but not on patient outcomes, and the effectiveness of PEMs compared to other interventions is uncertain (Farmer 2008).

Advances in electronic technologies have made health information more available. For example, databases on CD-ROM, the Internet and personal digital assistant (PDAs) enable healthcare professionals to access medical information electronically in seconds. Electronic resources contain a range of information from primary studies that can be found in journals to synthesized sources including electronic books and synopses of research-based publications (for example, Clinical Evidence ${ }^{\circledR}$ ).

The Internet is a convenient way to access other types of electronic health information. Healthcare professionals can search for primary research in bibliographic databases such as MEDLINE, or for critically appraised and synthesized information in searchable databases such as The Cochrane Library and ACP Journal Club. Guidelines are available on the Internet and searchable via clearinghouses (for example, the National Guidelines Clearinghouse). To help healthcare professionals cope with information overload, many web sites provide 'one-stop shopping', with links to medical information resources in one place (such as the UK National Library for Health) or links to journals, guidelines and textbooks within one search (such as the TRIP Database at www.tripdatabase.com and InfoClinique at http:// infoclinique.fmed.ulaval.ca).

Many healthcare professionals have ready access to the Internet. For example, the 2007 Canadian National Physician Survey (NPS) found that only $14.7 \%$ of physicians did not have access to the Internet (NPS 2007). As well, there most nurses and allied health professionals in the UK, US, Canada and New Zealand (Gilmour 2008; Judd 2004; Kirk 2001; Nursing Standard 2002) have access to the Internet. However, there are gaps in access to electronic information resources, particularly in developing countries and rural areas. In sub-Saharan Africa, freely available digital information resources are under-utilized by health information professionals (Ajuwon 2008). Moreover, the inequity in online information access between professionals in rural health and other health settings has led to arguments for outreach efforts towards these underserved practitioners (Dorsch 2000). In African countries, textbooks remain the main source of information for postgraduate doctors in training, although researchers tend to prefer online access (Smith 2007).

Does access to electronic health information, as opposed to traditional sources of information such as printed textbooks, improve provider practice and health outcomes for patients? We completed a systematic review of interventions intended to provide electronic access to health information for healthcare practitioners to improve patient care. We looked at access to information in terms of information retrieval (active access to information). In line with the generic 'Acquisition - Cognition - Application' model proposed by Saracevic and Kantor: (1) health professionals retrieve information related to their intentions (acquisition); (2) they absorb, understand and integrate retrieved hits (cognition); and (3) they may use this newly understood and cognitively processed information (application) (Saracevic 1997).

\section{O B J E C T IVES}

To estimate the effectiveness of electronic retrieval of health information to healthcare providers.

\section{We considered the following comparisons.}

- Electronic retrieval of information compared to no electronic retrieval (or no intervention) in practice.

- Electronic retrieval of information compared to access to print based materials only.

- Electronic retrieval of information compared to one or more other types of electronic retrieval of information.

- Enhanced electronic retrieval of information compared to access to the electronic resource as part of standard practice.

We considered any objective measure of professional behavior (interventions that influence a professional such as specialist referrals, academic detailing, etc.) or patient outcome (for example, length of hospital stay).

\section{METHODS}

\section{Criteria for considering studies for this review}

\section{Types of studies}

Randomized controlled trials (RCTs) (including cluster randomized trials (CRCTs)), controlled clinical trials (CCT) and interrupted time series analyses (ITS) in which there was a clearly defined point in time when the intervention occurred and at least three data points before and three after the intervention. We included studies regardless of language of publication or publication status.

\section{Types of participants}

We considered healthcare providers, including physicians, nurses and allied healthcare professions (such as physiotherapists, speech pathologists, social workers, etc.) involved in providing direct patient care eligible. We did not consider allied health professionals not involved with direct patient care (for example, administrators, data analysts) and students for this review. 


\section{Types of interventions}

We considered the following interventions.

- Provision or increased access to electronically retrievable information (such as free access to particular journals or databases).

- Provision of electronically retrievable information at point of patient care delivery or elsewhere in the workplace (for example, library, offices). What was made available for retrievable access needed to be described in both the intervention and control groups.

- Training component where there was differential provision of electronically retrievable information between groups.

The broader term 'health' was used to mean any type of medical, nursing or allied health information and the term information was used interchangeably for information or knowledge. Medical knowledge has been defined as "information about diseases, therapies, interpretation of lab tests, etc, which is potentially applicable to decisions about multiple patients and public health policies" (Wyatt 2002)

We defined information retrieval to include active methods used to search for and identify information. An example would be the search and retrieval of information stored on a database or on the Internet, based on specified criteria (Westbrook 2005). The technologies included Internet-based access, CD-ROM based access, and PDAs to retrieve health information. The review included accessing electronic information, which is equivalent to consulting a textbook. This review did not include interventions pushing health information to users or interventions requiring prompting, such as alerts or reminders. We did not review clinical decision support systems or patient-related data systems which are used for patient specific advice (for example, Ottawa Ankle Rule).

\section{Types of outcome measures}

We included any objective or blind measure of professional behavior (for example, specialist referrals, academic detailing) or patient outcome (for example, length of hospital stay). We collected measures of health practitioners' knowledge, attitudes or satisfaction, the costs of the intervention (both its provision and implementation) and adverse events as secondary outcomes. We excluded studies reporting knowledge, satisfaction or attitudes alone in absence of the above-noted measures.

\section{Search methods for identification of studies}

We searched the following electronic databases.

(a) The EPOC Register (and the database of studies awaiting assessment).

(b) Cochrane Central Register of Controlled Trials (CENTRAL) (The Cochrane Library, Issue 3, 2008)

(c) MEDLINE (1966 - July 2008), AMED (Allied and Complementary Medicine) (1985 - July 2008), CAB Health (1973 - June 2004), CINAHL (1981-July 2008), Embase (1980 - week 38 2008), ERIC (Educational Resources Information Center) (1966 - October 2007), LILACS (the Latin American and Caribbean Literature on Health Sciences Database) (1982 - July 2008) and LISA (Library and Information Science Abstracts) (1969 - July 2008).

\section{Other sources}

(a) Reference lists of all papers and relevant reviews identified and selected review of reference lists from cited papers (through Web of Knowledge, July 2008).

(b) We contacted authors of relevant papers regarding any further published or unpublished work.

(c) We contacted organizations (such as aid agencies, access providers) working with developing countries regarding relevant studies of which they might be aware.

We developed a MEDLINE search strategy (Appendix 1) using the methodological component of the EPOC search strategy combined with selected MeSH terms and free text terms, and then validated and tested this. We translated the search string into the other databases using the appropriate controlled vocabulary as applicable (Appendix 2).

\section{Data collection and analysis}

We used the following methods in updating this review.

\section{Screening}

We retrieved the title and abstract of all records identified by electronic searches for relevancy screening and downloaded to a bibliographic software program (Reference Manager $($ ) ) and removed duplicates. We then uploaded these records to an Internet-based, secured, systematic review software program. Seven review authors independently screened all titles and abstracts to assess which studies met the inclusion criteria (with two review authors examining each title). We resolved any disagreement by discussion among the review authors $(K D, K H$, RG, ML, PP, JM, VW). We retrieved full text copies of all potentially relevant papers. We selected studies for data abstraction after two review authors had assessed them as eligible.

\section{Data abstraction}

Four review authors undertook data abstraction independently using a standardized electronic tailored data collection form based on the generic EPOC data collection checklist (KD, KH, RG and JM), with two review authors abstracting each study. We resolved any disagreement by discussion or with an arbitrator (EPOC review editor).

\section{Quality}

Four review authors independently assessed the methodological quality of all included studies (KD, KH, RG, JM), with two authors per study using criteria described in the EPOC module (http:// www.epoc.uottawa.ca). We resolved discrepancies by discussion. Two review authors undertook the risk of bias assessment (JM, KD).

\section{Reporting}

For each study, data are reported in natural units. Where baseline data are available from RCTs, CCTs and CBAs, we have reported pre-intervention and post-intervention means, proportions or percentages for both study and control groups.

\section{Analytical approach}

If a primary measure was not identified in the study, we calculated effects sizes for all reported outcomes and used the median effect size to represent the study. 


\section{RESULTS}

\section{Description of studies}

We identified 5,070 potentially relevant studies through database searches, reference lists and contacting authors (Figure 1). Following a process to locate duplicate citations, we removed 1,635 citations that were considered to be duplicate references to a record already retrieved. One hundred and twenty-seven which we screened again with a focus on design. We excluded103 studies based on ineligible study designs. This resulted in 24 references that we reviewed further for preliminary data extraction. However after detailed assessment, we excluded 20 of these. We identified one ongoing study. Of the three references left, two are studies that we have included in this review; the third is a protocol to one of the included studies. 
Figure 1.

\section{Figure 1 - PRISMA Flow Diagram}

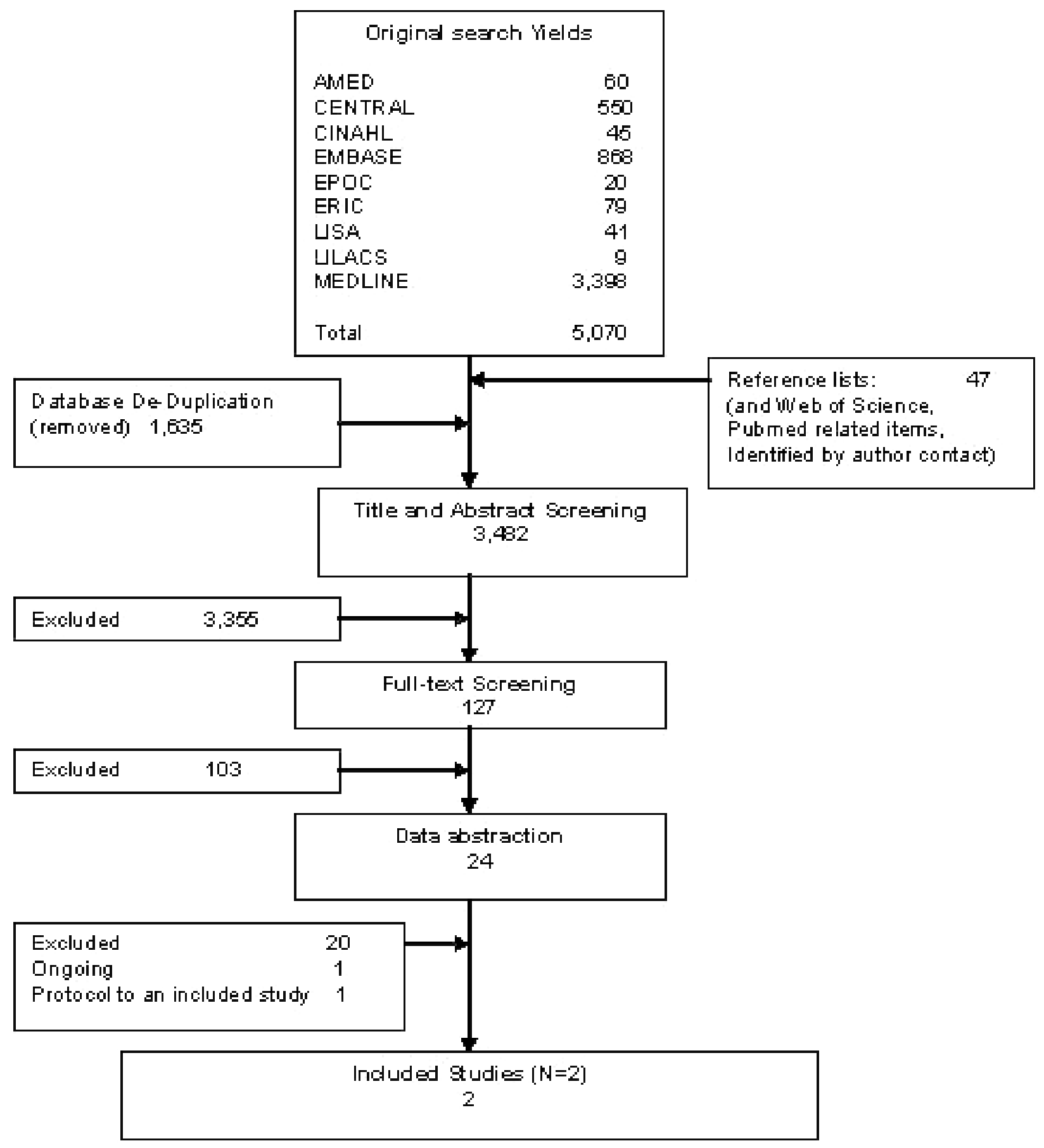

\section{Excluded studies}

Of the 20 excluded studies, six did not meet EPOC study design criteria (Deurenberg 2008; Dykes 2005; Gulmezoglu 1997; Rudin 1996; Rudin 1997; Sintchenko 2004); one did not assess active retrieval of information (Stewart 2005); two did not use objective assessment of behavior change (Alper 2005; Bullard 2004); and six assessed only knowledge outcomes (Butzlaff 2004; Casebeer 2003; D'Alessandro 2004; Elhadad 2005; Grad 2005; Southard 2003). 
Two studies did not target healthcare professionals (Di 2003; Kronick 2003); and one (Wyatt 1998) compared access to electronic materials as part of a multi-faceted intervention. In this one, however, there was no measure of information retrieval that could be used and there were issues of contamination in the control group. One study did not include participants that were directly involved in direct patient care (Forsetlund 2003).

\section{Ongoing studies}

One study (Coiera 2006) is a protocol for an RCT (see Characteristics of ongoing studies). In this study, participants have been randomized to receive access to receive an online evidence retrieval system in their practice for 12 months; the primary end-point is clinician acceptance and use of an online evidence retrieval system, and the resulting change in decision-making behavior.

\section{Included studies}

This review includes two studies, both CRCTs (Gulmezoglu 2007; Jousimaa 2002). One study was conducted in Thailand and Mexico (Gulmezoglu 2007), and the other in Finland (Jousimaa 2002); one had a duration of one month (Jousimaa 2002), and the other of one year (Gulmezoglu 2007).

\section{Characteristics of setting and professionals}

One study dealt with family physicians (Jousimaa 2002). One focused on educating nurses and midwives and physicians involved in obstetric practice (Gulmezoglu 2007). One did not report on gender (Gulmezoglu 2007), and the other was primarily female (\% female: Intervention Group (IG): 69.4; Control Group (CG): 73.1) (Jousimaa 2002). Age was not reported in one study (Gulmezoglu 2007), while the median age of the other study was early twenties (Jousimaa 2002).

\section{Characteristics of intervention}

Both studies (Gulmezoglu 2007; Jousimaa 2002) looked at the provision of access to electronically retrievable information. One study looked at the Cochrane Reproductive Health Library (RHL) (Gulmezoglu 2007) and the other study looked at national family practice guidelines in Finland (Jousimaa 2002). One study included a training component (Gulmezoglu 2007).

\section{Primary outcome measures}

We included studies that reported objective or blind measure of professional behavior or patient outcomes. One study (Jousimaa 2002) measured physicians' compliance with guideline recommendations (including laboratory, radiological, physical and other examinations, procedures, non-pharmacologic and pharmacologic treatments, physiotherapy and referrals). The other(Gulmezoglu 2007) looked at changes in 10 selected clinical practices (including social support during labour) as recommended by the Cochrane Reproductive Health Library (RHL). Neither study reported any measures of patient outcomes.

\section{Secondary outcome measures}

One study (Gulmezoglu 2007) measured knowledge as a secondary outcome. Neither study reported the costs of the provision of the electronic resource or the implementation of the recommended evidence-based practices. Neither study reported any adverse events.

\section{Risk of bias in included studies}

We assessed risk of bias for all studies using The Cochrane Collaboration's assessment tool. The components of the tool examine sequence generation; allocation concealment; blinding of participants, personnel, and outcome assessors; incomplete outcome data; and selective outcome reporting. Two authors (JM, $\mathrm{KD}$ ) independently assessed each study and provided text directly from the original report for which the assessment was derived. The authors reached consensus on assessment.

Adequate sequence generation and allocation concealment was reported in both studies (Gulmezoglu 2007; Jousimaa 2002). Blinding of outcome assessors was present in one study (Gulmezoglu 2007). Issues relating to incomplete outcome data were addressed in one study (Jousimaa 2002). Both studies were free of selective reporting (Gulmezoglu 2007; Jousimaa 2002). Overall, the information from both studies is at low risk of bias.

\section{Effects of interventions}

\section{Comparison group 1: Electronic retrieval of information compared to no electronic retrieval (or no intervention) in practice setting}

No studies assessed this comparison.

\section{Comparison group 2: Electronic retrieval of information compared to access to printed educational based materials only}

One study addressed this comparison: Jousimaa 2002 compared access to a computerized versus text based versions of the Evidence-Based Medicine Guidelines (EBMG). No statistically significant differences in physician consultation practices were found for any of the measured outcomes (Table 1).

\section{Comparison group 3: Electronic retrieval of information compared to other type of electronic retrieval of information}

No studies assessed this comparison.

\section{Comparison group 4: Enhanced electronic retrieval of information compared to access to the electronic resource as part of standard practice}

One study addressed this comparison: Gulmezoglu 2007 used a multifaceted intervention based on the Cochrane Reproductive Health Library $(R H L)$, which also addressed potential barriers to the implementation of evidence-based practices in the intervention group hospitals. Interactive workshops comprised the central activity of the intervention. The standard practice group did not receive any training, but had access to the RHL. The rates of compliance with ten targeted practices improved in both the intervention and control groups in both countries. There were no significant differences in rates of compliance with guidelines between the groups (Table 1). Knowledge of the RHL and its use increased in Mexico from $24.8 \%$ (78/314) to $65.5 \%$ (210/307) and $33.5 \%(65 / 194)$ to $39.2 \%(62 / 158)$ in the intervention and control groups, respectively. In Thailand, knowledge of RHL was 33.9\% $(57 / 168)$ and $38.2 \%(58 / 152)$ in the intervention and control groups at baseline. In the intervention group, knowledge of RHL increased to $83.3 \%$ (120/144), but comparable data were missing for the control group. 


\section{DISCUSSION}

This review focused on electronic retrieval of health information to healthcare providers. We searched for studies that could provide reliable evidence of the size and direction of effect of interventions that improved access to electronic resources by healthcare professionals. This meant that we limited our study designs to RCT, CRCT, CCT, and ITS, as these designs have the potential to provide reliable evidence. We also wanted to ensure that the intervention had impact on the behavior of healthcare professionals and, ideally, on patient outcomes.

We found two studies (Gulmezoglu 2007; Jousimaa 2002) which were heterogeneous in the style of intervention and the outcomes reported so they could not be combined in a meta-analysis. Neither of the studies found any changes in professional behavior after the provision of an intervention that facilitated electronic retrieval of health information. There was some evidence of improvements in knowledge about the electronic sources of information in one study (Gulmezoglu 2007). Neither of the studies assessed changes in patient outcomes or the costs of provision of the electronic resource and the implementation of the recommended evidencebased practices.

\section{Individual studies}

Gulmezoglu 2007:

The study was missing some items of data about information that would allow readers to assess the risk of bias (for example, method of randomization and concealment of allocation). This information was subsequently gained from personal communication with the authors.

The background level of access to the Cochrane Reproductive Health Library (RHL) was relatively high in this study, but it reflects 'standard practice' in this setting. The impact of the educational visit appears to improve the rates of use of $\mathrm{RHL}$, but did not have direct impact on physician behavior as measured by ten index practices. The authors of this study felt that their focus on 'knowledge access', instead of targeting one or two interventions, may have decreased the chances of a positive effect on specific practice changes. The format of Cochrane reviews may have created a barrier to its message being used in practice (Badgett 2008). It may be that busy clinicians, nurses and midwives have difficulty putting the evidence into practice from a number of reviews rather than evidence-based guidelines, which allow a number of Cochrane recommendations to be placed into practical context. Clinicians tend to want quick, prescriptive advice at the bedside (Ely 2005).

\section{Jousimaa 2002:}

This study did not identify any significant differences in physicians' consultation practices in any of the measured outcomes between the computerized and textbook guidelines groups. There may have been ceiling effects in the rates of compliance with guidelines as the majority of these were very high in the control group (average $88 \%$ compliance). The physicians recruited to this study were all recent graduates, and it could be argued that they were more likely (than experienced physicians) to consult and comply with current guidelines, as they were less likely to have experience of alternative practices. The authors of this study indicated that other factors should be considered when choosing the method of presentation of guidelines, such as information retrieval times, ease of use during the consultation, ability to update, production costs and physicians' preferences. They also indicated that implementation of computerized guidelines may need more training and investment but when computers are readily available and routinely used within consultations, the computerized version offers many advantages such as easy updating, low production costs and the possibility to include other databases.

\section{Design issues}

\section{Intervention group}

Few would argue that access to and use of information by healthcare providers is not useful and not needed to practice evidence-based medicine (Sackett 2007). There are many factors that influence the use of electronic information, including quality (for example, validity), usefulness (for example, situational relevance for clinicians), and socio-technical issues (for example, accessibility at the point-of-care). Other factors include how the information is presented (for example, interactive screens or simply text based), how the search engine is structured, the credibility of the source, where the information is stored (for example, through a library, commercial sites), and time required to read, process and apply the information. How information is presented is important. As time pressure limits the use of electronic retrieval of health information in clinical practice, healthcare professionals can be encouraged to increase their use of summaries through existing digital libraries (Haynes 2006) and to answer more clinical questions by using synthesized evidence (Alper 2005).

\section{Control group 'contamination'}

There are obvious difficulties when trying to determine the size of the effect of any intervention when the control group has some access to the same electronic resources as part of standard practice. However, this is pragmatically the only way these sorts of trials can be done in the real world. Concern about control group contamination is one of the major considerations for choosing the cluster randomization design since there is the possibility of an intervention leaking from one colleague to another within the same unit.

\section{Outcomes}

Patient outcomes: neither of the identified studies measured the impact of the interventions on patient outcomes. It is vital that future trials include these outcomes or we cannot be sure that any changes in behavior in the healthcare professionals are having the desired effect on patient care and outcomes. However, this may require longer follow-up to detect changes in patient outcomes and larger sample sizes.

Costs: neither study measured the costs of the interventions, nor the cost of implementing the changes in practice that would result from closer adherence to evidence-based recommendations.

Adverse effects: neither study reported any adverse effects as a result of bringing in improved access to electronic healthcare information. Theoretically these could include the following issues: 1. increased time to find the information; 2 . patients may find the doctors consulting a computer impersonal; or 3 . it may reduce patient perception or trust in the outcome of the consultation. 
Future studies of electronic retrieval of health information to healthcare providers may benefit from interventions designed to evaluate health professional behavior and practice changes as well as patient-related outcomes, as we found only two studies meeting our criteria.

Some may argue that it would still be relevant to conduct further research comparing electronic retrieval methods to other 'traditional' methods. However, as it is likely that electronic methods are here to stay and have some advantages over traditional methods, research that focuses on comparing differing methods of access to electronic information may make more sense.

\section{Potential limitations of the review}

Our review does have limitations. We only found two studies of uncertain generalizability, as they were conducted in very specific settings where there was already a high level of access to electronic resources. Additionally, we may have missed some studies due to poor labelling in bibliographic databases. For example, in our Medline and CINAHL strategies (Appendix 1; Appendix 2) we were not able to find indexing terms specific to our question. As such, we used a wide variety of indexing terms and text word terms (terms that search in the title and abstract of a bibliographic record) to try to account for the different ways in which indexers may have indexed the studies or the ways that study authors may have described their studies. This meant that we had a very low specificity in our search results (for example, our strategy retrieved a large number of citations with few that were relevant).

From a learning perspective, accessing information is important for clinicians, not only for supporting changes in clinical practice, but also for confirming their current practice. However, we did not take into account, by design, other potential benefits associated with information technologies with respect to physicians' continuing professional development, specifically the possibility for easily confirming that what you are doing is still correct in accordance with the health literature.

\section{AUTHORS' CONCLUSIONS}

\section{Implications for practice}

- This review found only two studies that examined the use of electronic retrieval of healthcare information by healthcare providers to improve practice and patient care. The results of these studies had low risk of bias but could not be combined in a meta-analysis. Neither study found evidence that electronic retrieval of healthcare information changed professional behavior; one study found that knowledge was improved.

- Overall there was insufficient evidence to support or refute the use of electronic retrieval of healthcare information by healthcare providers to improve practice and patient care. Pragmatically, access to electronic information may be beneficial to the practice of evidence-based health care, but appears to be insufficient in itself to influence behavior change in healthcare professionals.

- It is likely that access to electronic information is beneficial to the practice of evidence-based health care, but by itself, insufficient to improve practice and patient care.

\section{Implications for research}

- Future studies should attempt to strengthen the evidential links between changes in clinician's knowledge and behaviors, with patient outcomes and costs.

- Future studies should evaluate the rate at which the electronic resources are used by both the intervention and control groups.

- The acceptability to patients of healthcare professionals using computer resources during consultations should be examined.

- The type of electronic information available (papers, reviews, guidelines, synopses); how it is accessed (styles of interface); where (at the bedside); and when (during consultations or after) are all important variables to be considered in future research.

- Future studies should report their findings with care and report all of the information necessary for a full assessment of the risks of bias and of the results. Adherence to guidelines such as the CONSORT guidelines would aid this effort (Moher 2001).

\section{ACKNOWLEDGEMENTS}

Prof Martin Eccles provided invaluable advice and assistance throughout the development of this review. We would also like to thank Laura McAuley, Martin Dawes, Maria Judd, Yvan Leduc and George Wells, who contributed to the development of the protocol for this review. We would also like to thank Andrew Booth, Luis Gabriel Cuervo, Manisha Pahwa, Maria Claude Lavoie for their helpful comments on this review. 


\section{RE F E R E N C E S}

\section{References to studies included in this review}

Gulmezoglu 2007 \{published data only\}

* Gulmezoglu AM, Langer A, Piaggio G, Lumbiganon P, Villar J, Grimshaw J. Cluster randomised trial of an active, multifaceted educational intervention based on the WHO Reproductive Health Library to improve obstetric practices. BJOG: an international journal of obstetrics and gynaecology 2007;114(1):16-23. [PUBMED: 17010115]

Gulmezoglu AM, Villar J, Grimshaw J, Piaggio G, Lumbiganon P, Langer $A$. Cluster randomized trial of an active, multifaceted information dissemination intervention based on The WHO Reproductive Health Library to change obstetric practices: methods and design issues [ISRCTN14055385].. BMC Medical Research Methodology 2004;4(1):2. [PUBMED: PubMed: 14723792]

ISRCTN14055385. A randomised controlled trial to evaluate a programme promoting evidence-based medicine based on the World Health Organization (WHO) Reproductive Health Library. http://www.controlled-trials.com/ISRCTN14055385/14055385 (assessed 23 September 2008).

\section{Jousimaa 2002 \{published data only\}}

Jousimaa J, Makela M, Kunnamo I, MacLennan G, Grimshaw JM. Primary care guidelines on consultation practices: the effectiveness of computerized versus paper-based versions. A cluster randomized controlled trial among newly qualified primary care physicians. International Journal of Technology Assessment in Health Care 2002;18(3):586-96. [PUBMED: 12391951]

\section{References to studies excluded from this review}

\section{Alper 2005 \{published data only\}}

Alper BS, White DS, Ge B. Physicians answer more clinical questions and change clinical decisions more often with synthesized evidence: a randomized trial in primary care. Annals of Family Medicine 2005;3(6):507-13. [PUBMED: 16338914]

\section{Bullard 2004 \{published data only\}}

Bullard MJ, Meurer DP, Colman I, Holroyd BR, Rowe BH. Supporting clinical practice at the bedside using wireless technology. Academic Emergency Medicine 2004;11(11):1186-92. [PUBMED: 15528583]

\section{Butzlaff 2004 \{published data only\}}

Butzlaff M, Vollmar HC, Floer B, Koneczny N, Isfort J, Lange S. Learning with computerized guidelines in general practice?: a randomized controlled trial. Family Practice 2004;21(2):183-8. [PUBMED: 15020389]

\section{Casebeer 2003 \{published data only\}}

Casebeer LL, Strasser SM, Spettell CM, Wall TC, Weissman N, Ray MN, et al. Designing tailored web-based instruction to improve practicing physicians' preventive practices. Journal of Medical Internet Research 2003;5(3):e20. [PUBMED: 14517111]
D'Alessandro 2004 \{published data only\}

D'Alessandro DM, Kreiter CD, Peterson MW. An evaluation of information-seeking behaviors of general pediatricians. Pediatrics 2004;113(1 Pt 1):64-9. [PUBMED: 14702450]

Deurenberg 2008 \{published data only\}

Deurenberg R, Vlayen J, Guillo S, Oliver TK, Fervers B, Burgers J. Standardization of search methods for guideline development: an international survey of evidence-based guideline development groups. Health Information and Libraries Journal 2008;25(1):23-30. [PUBMED: 18251909]

\section{Di 2003 \{published data only\}}

Di Noia J, Schwinn TM, Dastur ZA, Schinke SP. The relative efficacy of pamphlets, CD-ROM, and the Internet for disseminating adolescent drug abuse prevention programs: an exploratory study. Preventive Medicine 2003;37(6 Pt 1):646-53. [PUBMED: 14636798]

\section{Dykes 2005 \{published data only\}}

Dykes PC, Acevedo K, Boldrighini J, Boucher C, Frumento K, Gray $P$, et al. Clinical practice guideline adherence before and after implementation of the HEARTFELT (HEART Failure Effectiveness \& Leadership Team) intervention. Journal of Cardiovascular Nursing 2005;20(5):306-14. [PUBMED: 16141775]

Elhadad 2005 \{published data only\}

Elhadad N, McKeown K, Kaufman D, Jordan D. Facilitating physicians' access to information via tailored text summarization. AMIA Annual Symposium proceedings. 2005:226-30. [PUBMED: 16779035]

\section{Forsetlund 2003 \{published data only\}}

Forsetlund L, Bradley P, Forsen L, Nordheim L, Jamtvedt G, Bjorndal A. Randomised controlled trial of a theoretically grounded tailored intervention to diffuse evidence-based public health practice [ISRCTN23257060]. BMC Medical Education 2003;3:2. [PUBMED: 12694632]

ISRCTN23257060. Towards evidence-based public health http://www.controlled-trials.com/ISRCTN23257060/23257060 (assessed 23 September 2008).

\section{Grad 2005 \{published data only\}}

Grad RM, Meng Y, Bartlett G, Dawes M, Pluye P, Boillat M, et al. Effect of a PDA-assisted evidence-based medicine course on knowledge of common clinical problems. Family Medicine 2005;37(10):734-40. [PUBMED: 16273453]

\section{Gulmezoglu 1997 \{published data only\}}

Gulmezoglu AM, Villar J. Providing access to evidence-based information in reproductive health: the WHO Reproductive Health Library for developing countries. Sao Paulo medical journal = Revista paulista de medicina 1997; Vol. 115, issue 4:1469-70. [PUBMED: 9595810]

Haynes 2006 \{published data only\}

Haynes RB, Holland J, Cotoi C, McKinlay RJ, Wilczynski NL, Walters LA, et al. McMaster PLUS: a cluster randomized clinical 
trial of an intervention to accelerate clinical use of evidencebased information from digital libraries. Journal of the American Medical Informatics Association 2006;13(6):593-600. [PUBMED: 16929034]

\section{Kronick 2003 \{published data only\}}

Kronick J, Blake C, Munoz E, Heilbrunn L, Dunikowski L, Milne WK. Improving on-line skills and knowledge. A randomized trial of teaching rural physicians to use on-line medical information. Canadian Family Physician/Medecin de Famille Canadien 2003;49:312-7. [PUBMED: 12675544]

\section{Rudin 1996 \{published data only\}}

Rudin JL. Combined internal medicine and pharmaceutical electronic reference resources: Part 1. Compendium of Continuing Education in Dentistry 1996;17(9):822-5;828-30. [PUBMED: 9161155]

\section{Rudin 1997 \{published data only\}}

Rudin JL. Combined internal medicine and pharmaceutical electronic reference resources: Part 2. Compendium of Continuing Education in Dentistry 1997;18(1):28-30; 32-4; 36-8. [PUBMED: 9161135]

\section{Sintchenko 2004 \{published data only\}}

Sintchenko V, Coiera E, Iredell JR, Gilbert GL. Comparative impact of guidelines, clinical data, and decision support on prescribing decisions: an interactive web experiment with simulated cases. Journal of the American Medical Informatics Association 2004;11(1):71-7. [PUBMED: 14527970]

\section{Southard 2003 \{published data only\}}

Southard BH, Southard DR, Nuckolls J. Clinical trial of an Internet-based case management system for secondary prevention of heart disease. Journal of Cardiopulmonary Rehabilitation 2003;23(5):341-8. [PUBMED: 14512778]

\section{Stewart 2005 \{published data only\}}

Stewart M, Marshall JN, Ostbye T, Feightner JW, Brown JB, Harris S, et al. Effectiveness of case-based on-line learning of evidence-based practice guidelines. Family Medicine 2005;37(2):131-8. [PUBMED: 15690254]

\section{Wyatt 1998 \{published data only\}}

Wyatt JC, Paterson-Brown S, Johanson R, Altman DG, Bradburn MJ, Fisk NM. Randomised trial of educational visits to enhance use of systematic reviews in 25 obstetric units. BMJ (Clinical research ed.) 1998;317(7165):1041-6. [PUBMED: 9774287]

\section{References to ongoing studies}

\section{Coiera 2006 \{published data only\}}

Coiera E, Magrabi F, Westbrook JI, Kidd MR, Day RO. Protocol for the Quick Clinical study: a randomised controlled trial to assess the impact of an online evidence retrieval system on decision-making in general practice. BMC Medical Informatics and Decision Making 2006;6:33. [PUBMED: 16928282]

\section{Additional references}

\section{Ajuwon 2008}

Ajuwon GA, Rhine L. The level of Internet access and ICT training for health information professionals in sub-Saharan Africa. Health Information and Libraries Journal 2008;25(3):175-85. [PUBMED: 18796078]

\section{Badgett 2008}

Badgett R. Why would physicians undervalue reviews by The Cochrane Collaboration?. Journal of Clinical Epidemiology 2008; Vol. 61, issue 5:419-21. [PUBMED: 18394533]

\section{Dawes 2003}

Dawes M, Sampson Uchechukwu S. Knowledge management in clinical practice: a systematic review of information seeking behavior in physicians. International Journal of Medical Informatics 2003;71:9-15.

\section{Dorsch 2000}

Dorsch JL. Information needs of rural health professionals: a review of the literature. Bulletin of the Medical Library Association 2000;88(4):346-54. [PUBMED: 11055302]

\section{Ely 2005}

Ely JW, Osheroff JA, Chambliss ML, Ebell MH, Rosenbaum ME. Answering physicians' clinical questions: obstacles and potential solutions. Journal of the American Medical Informatics Association : JAMIA 2005;12(2):217-24. [PUBMED: 15561792]

\section{Farmer 2008}

Farmer AP, Legare F, Turcot L, Grimshaw J, Harvey E, McGowan JL, et al. Printed educational materials: effects on professional practice and health care outcomes. Cochrane Database of Systematic Reviews 2008, Issue 3. [DOI: 10.1002/14651858.CD004398.pub2; PUBMED: 18646106]

\section{Gilmour 2008}

Gilmour JA, Scott SD, Huntington N. Nurses and Internet health information: a questionnaire survey. Journal of Advanced Nursing 2008;61(1):19-28. [PUBMED: 18034820]

\section{Haynes 2006}

Haynes RB. Of studies, syntheses, synopses, summaries, and systems: the " $5 \mathrm{~S}$ " evolution of information services for evidence-based health care decisions. ACP Journal Club 2006; Vol. 145, issue 3:A8. [PUBMED: 17080967]

\section{Judd 2004}

Judd M. Examining the use of evidence-based resources among physiotherapists in Canada. [dissertation] Ottawa, ON: University of Ottawa. 2004.

\section{Kirk 2001}

Kirk SFL, Cade JE, Greenhalgh A. Dietitians and the Internet: dietitians embracing the new technology?. Journal of Human Nutrition and Dietetics 2001;14:477-84.

\section{Moher 2001}

Moher D, Schulz KF, Altman DG. The CONSORT statement: revised recommendations for improving the quality 
of reports of parallel-group randomised trials. Lancet 2001;357(9263):1191-4. [PUBMED: 11323066]

\section{NPS 2007}

The College of Family Physicians of Canada, Canadian Medical Association, The Royal College of Physicians and Surgeons of Canada. National Physician Survey (NPS). http:// www.nationalphysiciansurvey.ca/nps/2007_Survey/2007npse.asp 2007.

\section{Nursing Standard 2002}

2002 RCN Publishing Company Internet Survey. http:// www.nursing-standard.co.uk/advertising/adpics/ ns_co_uk_media.pdf accessed 01 October 2003.

\section{Pakenham-Walsh 1997}

Pakenham-Walsh N, Priestly C, Smith R. Meeting the information needs of health workers in developing countries. BMJ (Clinical research ed.) 1997; Vol. 314, issue 7074:90. [PUBMED: 9006454]

\section{Pakenham-Walsh 2002}

Pakenham-Walsh N, Priestley C. Towards equity in global health knowledge. QJM Monthly Journal of the Association of Physicians 2002;95(7):469-73. [PUBMED: 12096152]

\section{Sackett 2007}

Sackett DL, Straus SE, Richardson WS, Rosenberg W, Haynes RB. Evidence-based medicine: how to practice and teach EBM. 2nd Edition. London: Churchill-Livingstone, 2007.

\section{Saracevic 1997}

Saracevic T, Kantor PB. Studying the value of library and information services. Part II. Methodology and taxonomy. Journal of the American Society for Information Science 1997;48(6):543-63.

\section{Smith 2007}

Smith H, Bukirwa H, Mukasa O, Snell P, Adeh-Nsoh S, Mbuyita S, et al. Access to electronic health knowledge in five countries in Africa: a descriptive study. BMC Health Services Research 2007;7:72. [PUBMED: 17509132]

\section{Westbrook 2005}

Westbrook JI, Coiera EW, Gosling AS. Do online information retrieval systems help experienced clinicians answer clinical questions?. Journal of the American Medical Informatics Association 2005;12(3):315-21. [PUBMED: 15684126]

\section{Wyatt 2002}

Wyatt JC, Liu JL. Basic concepts in medical informatics. Journal of Epidemiology and Community Health 2002;56(11):808-812.

* Indicates the major publication for the study

\section{CHARACTERISTICS OF STUDIES}

Characteristics of included studies [ordered by study ID]

Gulmezoglu 2007

\begin{tabular}{ll}
\hline Methods & CRCT \\
\hline Participants & Total randomized (by hospital): Thailand 18; 9 (IG); 9 (CG); Mexico 22; 13 (IG); 9 (CG) \\
& Age: NR \\
& Sex (\% female): NR \\
& Providers: Physicians, midwives, interns and students \\
& Setting: Community hospitals with > 1,000 deliveries per year \\
Country:Mexico and Thailand
\end{tabular}

Interventions

Intervention: A multifaceted intervention based on using the Cochrane Reproductive Health Library $(\mathrm{RHL})$, which also addressed potential barriers to the implementation of evidence-based practices in the intervention group hospitals. Interactive workshops comprised the central activity of the intervention.

Comparison: No intervention

Duration: 1 year

Follow up: NR

EPOC: Distribution of educational materials; conference/educational meetings; provision of computers to access RHL, get management on board, local coordinator

Outcomes

Primary outcome: Changes in the practice rates of 10 selected clinical practices (selected practices ranged from changes such as prescribing antibiotics to women at cesarean section to those that required organizational change within the units) as recommended in the RHL 
Gulmezoglu 2007 (Continued)

Secondary outcome: Knowledge of the Cochrane RHL

Notes Targeted behavior: clinical prevention services; general management of a problem/treatment

\section{Risk of bias}

\begin{tabular}{lll}
\hline Bias & Authors' judgement & Support for judgement \\
\hline $\begin{array}{l}\text { Adequate sequence gener- } \\
\text { ation? }\end{array}$ & Low risk & $\begin{array}{l}\text { Quote: "The stratified allocation was based on country, type of hospital and } \\
\text { number of births per year. The random allocation sequence was produced } \\
\text { centrally by WHO." Additional contact with authors provided further informa- } \\
\text { tion that this was done using PROC PLAN of SAS software. }\end{array}$ \\
\hline Allocation concealment? & Low risk & $\begin{array}{l}\text { Quote: "Country investigators were informed of the allocation status of the } \\
\text { hospitals after collection of baseline data was completed and when the first } \\
\text { workshop had to be organized as required by the protocol." Additional contact } \\
\text { with authors provided further information that the randomization occurred at } \\
\text { one time point, and allocation was concealed until after this time point. }\end{array}$ \\
\hline
\end{tabular}

Blinding? High risk The trial was not blinded.

All outcomes

Incomplete outcome data High risk The Thai control group data on process outcomes was missing.

addressed?

All outcomes

Free of selective report- Low risk
ing?
ing?

Primary, expected outcomes reported from published protocol. ISRCTN 14055385: (http://www.controlled-trials.com/ISRCTN14055385/14055385).

Jousimaa 2002

\begin{tabular}{ll}
\hline Methods & CRCT \\
\hline Participants & Total randomized: 139; IG: 72; CG 67 \\
& Age (mean): IG: 27.3; CG 26.9 \\
& Sex (\% female): IG: 69.4; CG: 73.1 \\
& Providers: Physicians (recently qualified) \\
& Setting: Primary health centres \\
& Country:Finland \\
\hline Interventions & Intervention: Physicians were randomized to receive a computerized version of the Evidence-Based \\
& Medicine Guidelines (EBMG) \\
& Comparison: Physicians were randomized to receive textbook based version of the Evidence-Based \\
& Medicine Guidelines (EBMG) \\
& Duration: 1 month \\
Follow up: NR & EPOC: Distribution of educational materials; other - paper versus computerized (CD-ROM) guidelines \\
\hline Outcomes & $\begin{array}{l}\text { Primary outcome: Physicians compliance with guideline recommendations (including laboratory, radi- } \\
\text { ological, physical and other examinations, procedures, nonpharmacologic and pharmacologic treat- } \\
\text { ments, physiotherapy and referrals). }\end{array}$ \\
\hline Notes & $\begin{array}{l}\text { Targeted behavior: clinical prevention services; diagnosis; test ordering; referrals; general management } \\
\text { of a problem/treatment; procedures; prescribing }\end{array}$ \\
\hline & \\
\hline &
\end{tabular}


Jousimaa 2002 (Continued)

\section{Risk of bias}

\begin{tabular}{|c|c|c|}
\hline Bias & Authors' judgement & Support for judgement \\
\hline $\begin{array}{l}\text { Adequate sequence gener- } \\
\text { ation? }\end{array}$ & Low risk & $\begin{array}{l}\text { Quote: "Students agreeing to participate in the study were randomised cen- } \\
\text { trally using computer-generated numbers." Note: they entered the study after } \\
\text { they qualified as physicians. }\end{array}$ \\
\hline Allocation concealment? & Low risk & $\begin{array}{l}\text { Quote: "Students agreeing to participate in the study were randomised cen- } \\
\text { trally using computer-generated numbers." }\end{array}$ \\
\hline $\begin{array}{l}\text { Blinding? } \\
\text { All outcomes }\end{array}$ & Low risk & $\begin{array}{l}\text { Quote: "The anonymous patient records were then evaluated by one author } \\
\text { (JJ, experienced primary care physician) blinded to the study group." }\end{array}$ \\
\hline $\begin{array}{l}\text { Incomplete outcome data } \\
\text { addressed? } \\
\text { All outcomes }\end{array}$ & Low risk & $\begin{array}{l}\text { Quote: "Data on } 4,633 \text { patient encounters were abstracted, of which 3,484 } \\
\text { were suitable for further analysis." }\end{array}$ \\
\hline $\begin{array}{l}\text { Free of selective report- } \\
\text { ing? }\end{array}$ & Low risk & $\begin{array}{l}\text { The author supplied a reconstruction of the study plan and letters to the par- } \\
\text { ticipating physicians (Appendix 3). }\end{array}$ \\
\hline
\end{tabular}

$\mathrm{CG}=$ control group

$\mathrm{CRCT}=$ Cluster randomized controlled trial

IG = intervention group

$\mathrm{RHL}=$ Reproductive Health Library

NR $=$ not reported

Characteristics of excluded studies [ordered by study ID]

\begin{tabular}{ll}
\hline Study & Reason for exclusion \\
\hline Alper 2005 & Intervention: no objective assessment of behavior change \\
\hline Bullard 2004 & Intervention: no objective assessment of behavior change \\
\hline Butzlaff 2004 & Intervention: only assessed knowledge outcomes/no EPOC outcomes \\
\hline Casebeer 2003 & Intervention: only assessed knowledge outcomes/no EPOC outcomes \\
\hline D'Alessandro 2004 & Study design: study is not complete (the initial results are based on knowledge test) \\
\hline Deurenberg 2008 & Intervention: only assessed knowledge outcomes/no EPOC outcomes \\
\hline Di 2003 & Study design: semi-structured questionnaire \\
\hline Dykes 2005 & Population: not aimed at healthcare professionals in healthcare settings \\
\hline Elhadad 2005 & Study design: historical control only (no other control group) and no randomization \\
\hline Forsetlund 2003 & Intervention: only assessed knowledge outcomes/no EPOC outcomes \\
\hline Grad 2005 & Participants: public health physicians were not involved in direct patient care. \\
\hline
\end{tabular}




\begin{tabular}{ll}
\hline Study & Reason for exclusion \\
\hline Gulmezoglu 1997 & Study design: editorial \\
\hline Haynes 2006 & Intervention: outcomes addressed use of service/no EPOC outcomes \\
\hline Kronick 2003 & Population: not aimed at healthcare professionals in healthcare settings \\
\hline Rudin 1996 & Study design: narrative article \\
\hline Rudin 1997 & Study design: narrative article \\
\hline Sintchenko 2004 & \begin{tabular}{l} 
Study design: simulated cases \\
\hline Southard 2003
\end{tabular} \\
\hline Intervention: only assessed knowledge outcomes/no EPOC outcomes \\
\hline Intervention: does include retrieval access to information \\
\hline $\begin{array}{l}\text { Intervention: compared access to electronic materials as part of a multi-faceted intervention; how- } \\
\text { ever, there was no measure of information retrieval that could be used and there were issues of } \\
\text { contamination in the control group. The final decision on the exclusion of this study was deter- } \\
\text { mined through arbitration. }\end{array}$ \\
\hline
\end{tabular}

Characteristics of ongoing studies [ordered by study ID]

Coiera 2006

\begin{tabular}{ll}
\hline Trial name or title & $\begin{array}{l}\text { Quick Clinical study: a randomized controlled trial } \\
\text { to assess the impact of an online evidence retrieval system on } \\
\text { decision-making in general practice }\end{array}$ \\
\hline Methods & Randomized controlled parallel design \\
\hline Participants & 200 general practitioners in Australia \\
\hline Interventions & $\begin{array}{l}\text { Participants are randomized to receive access to QC in } \\
\text { their practice for } 12 \text { months. }\end{array}$ \\
\hline Outcomes & $\begin{array}{l}\text { The primary end-points for the study is clinician acceptance and use of QC and the resulting } \\
\text { change in decision-making behavior }\end{array}$ \\
\hline Starting date & $\begin{array}{l}\text { Recruitment commenced in October 2004, 203 GPs volunteered } \\
\text { to participate in the study and the trial formally } \\
\text { commenced in May 2005. }\end{array}$ \\
\hline Contact information & $\begin{array}{l}\text { Enrico Coiera: e.coiera@unsw.edu.au } \\
\text { Notes }\end{array}$ \\
\hline $\begin{array}{l}\text { The RCT will examine prescribing patterns related to frequently prescribed medications where } \\
\text { there has been a recent significant shift in recommendations regarding their use based upon new } \\
\text { evidence. }\end{array}$ \\
\hline
\end{tabular}

\section{ADDITIONAL TABLES}


Table 1. Summary of Results

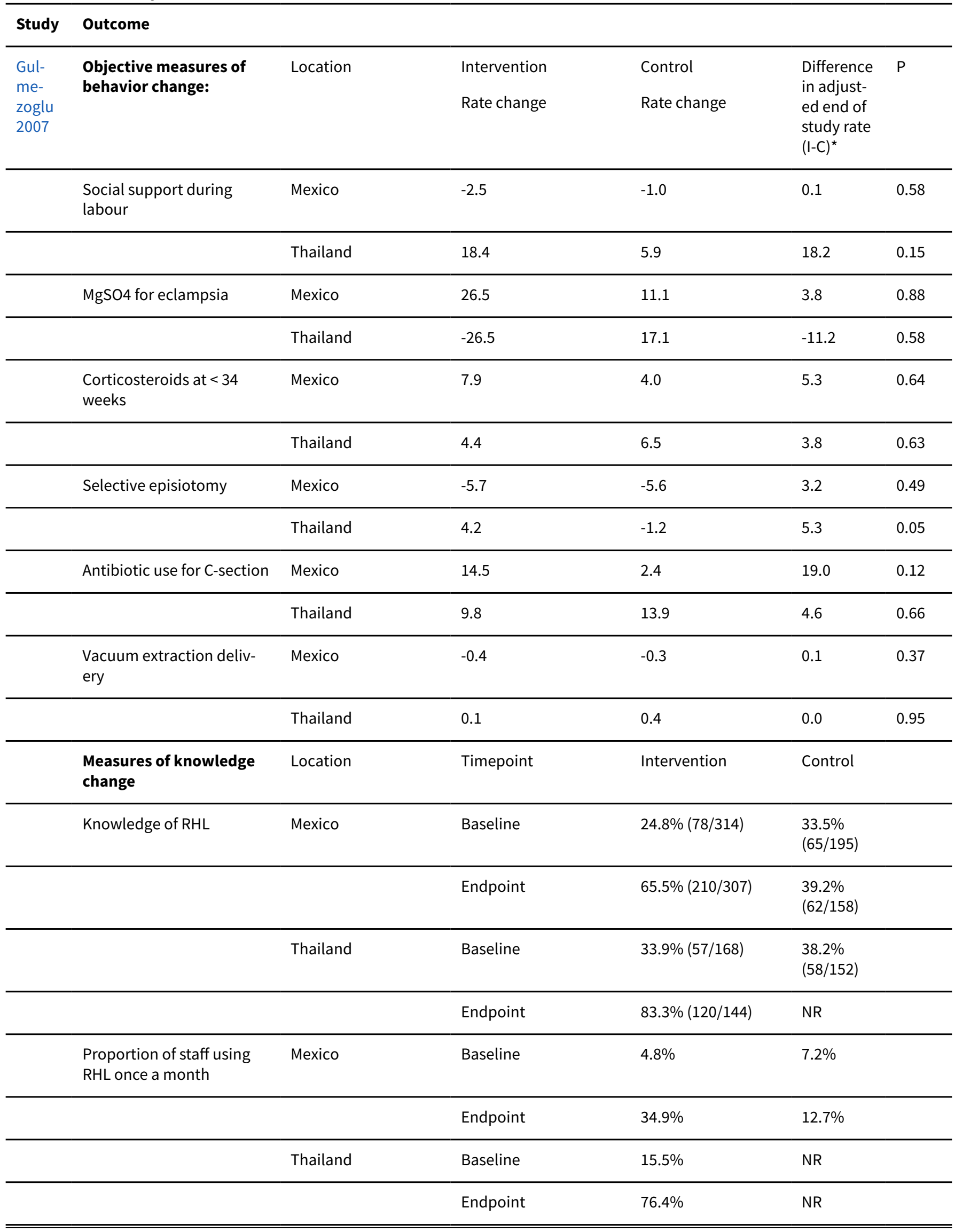


Table 1. Summary of Results (Continued)

\begin{tabular}{|c|c|c|c|c|c|}
\hline $\begin{array}{l}\text { Jousi- } \\
\text { maa } \\
2002\end{array}$ & $\begin{array}{l}\text { Objective measures of } \\
\text { behavior change: }\end{array}$ & $\begin{array}{l}\text { Intervention (\%: } \mathrm{n} \\
\text { guideline compliant } \\
\text { consultations/n rele- } \\
\text { vant consultations) }\end{array}$ & $\begin{array}{l}\text { Control (\%: } \mathrm{n} \text { guide- } \\
\text { line compliant con- } \\
\text { sultations/n relevant } \\
\text { consultations) }\end{array}$ & Odds Ratio $(95 \% \mathrm{Cl})$ & ICC \\
\hline & Laboratory examinations & $90.3 \%(1481 / 1640)$ & $89.7 \%(1372 / 1529)$ & $1.07(0.79,1.44)$ & 0.015 \\
\hline & Radiological examinations & $93.8 \%(1504 / 1604)$ & $93.3 \%(1416 / 1518)$ & $1.09(0.81,1.46)$ & 0 \\
\hline & Physical examinations & $92.8 \%(1494 / 1610)$ & $94.6 \%(1461 / 1545)$ & $0.74(0.51,1.06)$ & 0.015 \\
\hline & Other examinations & $74.8 \%(235 / 314)$ & $80.8 \%(248 / 307)$ & $0.71(0.43,1.36)$ & 0.021 \\
\hline & Procedures & $77.6 \%(152 / 196)$ & $81.9 \%(140 / 171)$ & $0.77(0.43,1.36)$ & 0 \\
\hline & Physiotherapy & $78.6 \%(77 / 98)$ & $80.6 \%(83 / 103)$ & $0.88(0.34,2.32)$ & 0.195 \\
\hline & $\begin{array}{l}\text { Nonpharmacologic treat- } \\
\text { ment }\end{array}$ & $87.0 \%(80 / 92)$ & $90.2 \%(110 / 122)$ & $0.73(0.22,2.41)$ & 0.058 \\
\hline & $\begin{array}{l}\text { Pharmacological treat- } \\
\text { ment }\end{array}$ & $84.1 \%(1391 / 1654)$ & $86.1 \%(1350 / 1568)$ & $0.85(0.67,1.09)$ & 0.010 \\
\hline & Referrals & $96.1 \%(1619 / 1684)$ & $95.6 \%(1508 / 1578)$ & $1.13(0.79,1.63)$ & 0.002 \\
\hline
\end{tabular}

*The differences reported are differences in the adjusted end of study rates between the intervention and the control groups, not differences in rate changes.

\section{A P P E N D I CES}

\section{Appendix 1. MEDLINE search strategy}

\section{MEDLINE via OVID}

1 exp online systems/

2 databases, bibliographic/ or databases, factual/

3 ((electronic or online or computerized) adj2 (access or retrieval or technolog\$)).tw.

4 exp compact disks/ or cd-rom/

5 Internet/ or Computers, Hand-Held/

6 (internet or CD-ROM or cd rom or compact disk\$).tw.

7 (www or world wide web).tw.

8 (pda or personal digital assistant\$ or handheld or hand held).tw.

9 or/1-8

10 exp manuals/ or exp reference books/ or textbooks/ or periodicals/

11 (textbook\$ or book\$ or journal\$ or periodical\$ or manual or manuals).tw.

12 exp Guidelines/

13 Information Dissemination/

14 or $/ 10-13$

159 and 14

16 databases, bibliographic/

17 (medline or pubmed or gratefulmed or embase or cinahl or cochrane or clinical evidence or mdconsult or inforetrieval).tw.

18 exp "information storage and retrieval"/ or medlars/

19 or/16-18

20 access $\$$.tw.

2119 and 20

2219 and 15 


\section{Appendix 2. CINAHL search strategy}

CINAHL (Cumulative Index to Nursing and Allied Health Literature) via OVID

1 exp online systems/

2 exp reference databases/

3 ((electronic or online or computerized) adj2 (access or retrieval or technolog\$)).tw.

4 optical disks/ or CD ROM/

5 Internet/ or Computers, Hand-Held/

6 (internet or CD-ROM or cd rom or compact disk\$).tw.

7 (www or world wide web).tw.

8 (pda or personal digital assistant\$ or handheld or hand held).tw.

9 or/ $1-8$

10 exp reference books/ or textbooks/ or serial publications/

11 (textbook\$ or book\$ or journal\$ or periodical\$ or manual or manuals).tw.

12 practice guidelines/

13 information management/ or exp information retrieval/

14 or $/ 10-13$

159 and 14

16 exp reference databases/

17 (medline or pubmed or gratefulmed or embase or cinahl or cochrane or clinical evidence or mdconsult or inforetrieval).tw.

18 exp information retrieval/ or medline/

19 or/16-18

20 access $\$$.tw.

2119 and 20

2219 and 15

\section{Appendix 3. Protocol for Jousimaa 2002}

Primary care guidelines on consultation practices: the effectiveness of computerized versus paper-based versions. A cluster randomized controlled trial among newly qualified primary care physicians.

OBJECTIVE: To compare the effects of computerized versus paper-based versions of the same guidelines on recently qualified physicians' consultation practices

METHODS: Two arm cluster randomized controlled trial. All physicians licensed in Finland in 1998 will be contacted by phone. Eligible physicians include those who will work at least two months in a health centre between the study period from February 1998 to September 1999. The physicians will be randomized by computer-generated randomization number to receive either computerized or textbook-based versions of the same guidelines for a 4-week study period. Prior to the study the physicians will have at least one month 's run-in period to get used to health centre work. Computers will be provided for the computer guideline group for the study period, if not available at workplace. Textbooks will be provided for the textbook guideline group. Physicians' compliance with guideline recommendations about laboratory, radiological, physical and other examinations, procedures, non-pharmacologic and pharmacologic treatments, physiotherapy, and referrals will be measured by case note review.

DATA ANALYSIS: Participating physicians are asked to identify, on a daily print-out of patient contacts, any consultation during which they have searched information to support patient care from any information source (information searching consultation). They are also asked to complete a brief questionnaire for each information search. Data will be collected for one month, or until a maximum of 50 information searching consultations are included.

The patient records are collected from information searching consultations and the preceeding consultations with a different patient which did not include information searches, and photocopied. Using this method, the physician will not know during the consultation, that the non-information seeking consultation is going to be analysed. All patient information data will be deleted from the photocopies in the health centre. The photocopies will be further mailed to an independent research centre, where the physician, health centre and study group will be anonymised. The anonymized record will then be evaluated by the three authors (JJ, IK, MM)* (SEE BELOW) and kappa statistics for concurrence will be calculated from a sample.

Nine elements will be evaluated: lab examinations, radiological examinations, physical examinations, other examinations for example, endoscopy, procedures, pharmacological treatments, non-pharmacological treatments, physiotherapy and referrals. Review criteria according to guidelines are developed for 99 commonest separate diagnoses, and the rest are evaluated case by case. Non-compliance with guidelines will be categorized as none, minor, major and serious.

STATISTICS. The physician is the unit of randomization and interference. The data will be analysed using adjusted Chi squared tests which account for the clustered nature of the data. 
Actually this never happened, as the others were too busy to do the job. So, the judgement whether the participant followed the guideline was solely up to $\mathrm{JJ}$, but the criteria for commonest diagnoses were pre-defined by three authors ( $\mathrm{JJ}$, IIK, MM) and these diagnoses covered over $80 \%$ of cases

\section{WHAT'S NEW}

\begin{tabular}{lll}
\hline Date & Event & Description \\
\hline 17 February 2010 & Amended & Change in author's contact details \\
\hline
\end{tabular}

\section{HISTORY}

Protocol first published: Issue 2, 2004

Review first published: Issue 3, 2009

\begin{tabular}{lll}
\hline Date & Event & Description \\
\hline 12 February 2004 & $\begin{array}{l}\text { New citation required and major } \\
\text { changes }\end{array}$ & Substantive amendment \\
\hline
\end{tabular}

\section{CONTRIBUTIONS OFAUTHORS}

Jessie McGowan drafted the protocol, and amended it with comments from all co-authors. Review authors screened studies, examined studies for eligibility, extracted data and analyzed the results. JM, RG, PP, and KD guided the data analysis planning and implementation. JM, RG, PP, KH, KD, ML, VW and PT assisted with interpretation of the results and clinical relevance.

\section{DECLARATIONS OF INTEREST}

None known.

\section{SOURCES OF SUPPORT}

\section{Internal sources}

- Institute of Population Health, University of Ottawa, Canada.

- Belgian Centre for Evidence-Based Medicine, Belgium.

- Department of Family Medicine, McGill University, Canada.

- Department of Family Medicine, Laval University, Canada.

\section{External sources}

- Canadian Institutes of Health Research, Post-doctoral Fellowship grant, Canada.

- Fonds de la recherche en santé du Québec, Canada.

- UK NHS Cochrane Collaboration Programme Grant Scheme, United Kingdom, Not specified.

\section{DIFFERENCES BETWEEN PROTOCOL AND REVIEW}

We further clarified that the type of access that we were focusing on in this review was 'electronic retrieval'. This is reflected in the title and objectives. We added in a definition of information retrieval in the background. We added the objectives: electronic retrieval of information compared to other types of electronic retrieval of information; and electronic retrieval of information compared to access to the electronic resource as part of standard practice. We added the outcomes costs and adverse events. 


\section{N D EX TERMS}

\section{Medical Subject Headings (MeSH)}

Evidence-Based Medicine [ ${ }^{*}$ statistics \& numerical data]; Guideline Adherence [statistics \& numerical data]; Health Personnel [ ${ }^{\star}$ statistics \& numerical data]; Patient Care; Professional Practice $\left[{ }^{\star}\right.$ standards]; Randomized Controlled Trials as Topic

\section{MeSH check words}

Humans 